\title{
Índice de Produção Científica Dos Docentes do Instituto Superior de Ciências de Educação do Huambo
}

\author{
Índice de Producción Científica de los Docentes del Instituto Superior \\ de Ciencias de la Educación de Huambo / Indices of Scientifics \\ Productions of the High Institute of Science Education in Huambo
}

João Baptista Machado Sousa*; Mário José da Costa Rodrigues**; Arlindo Emílio Joaquim Pedro***; Afonso Vindassi Manuel ${ }^{* * * *}$

\section{RESUMO}

A presente investigação é parte do relatório sobre a produção científica (PC) dos docentes do Instituto Superior de Ciências de Educação do Huambo no período de 2013 a 2017. O objectivo é de sistematizar a produção científica que o capital humano desta instituição tem vindo a desenvolver neste período. Considerou-se como indicadores para avaliar o Índice de Produção Científica (IPC), o número de artigos publicados em revistas científicas nacionais e internacionais, a participação em eventos científicos nacionais e internacionais e, finalmente, o número de livros e capítulos de livros publicados. Do ponto de vista metodológico, utilizou-se três instrumentos para a recolha de dados: um inquérito, um guia de entrevista e um guia para a análise documental com o qual se analisou os documentos existentes nos processos dos docentes, e a este último foi coadjuvado uma busca em diferentes bases de dados e motores na Internet e revistas citadas pelos inquiridos. No final, utilizou-se o método de triangulação de fontes, para contrastar as informações obtidas pelos instrumentos antes declarados. Como resultado desta análise, constatou-se 119 artigos científicos publicados, entre estes 75 em revistas nacionais e 44 internacionais; 189 participações em eventos científicos, das quais 113 nacionais e 76 internacionais, 7 livros publicados e, finalmente um capítulo de livro.

\footnotetext{
* Doutor em Ciências Pedagógicas, Universidade de Ciências Pedagógicas "Enrique José Varona"- Cuba. Chefe de Departamento de Investigação Científica e Pós-Graduação do Instituto Superior de Ciências de Educação de Huambo, Angola. Correio: sousangola@gmail.com

** Doutor em Ciências Pedagógicas, Universidade Marta Abreu de las Villas - Cuba. Director Geral do Instituto Superior de Ciências de Educação do Huambo, Angola. Correio: mariorodrigues_25@yahoo.com.br

*** Mestre em Biotecnologia, Instituto Superior Técnico de Lisboa - Portugal. Director Geral Adjunto Para Área Científica do Instituto Superior de Ciências de Educação do Huambo. Angola. Correio: ajepedro@ hotmail.com

${ }^{* * * * *}$ Mestre em Didáctica do Ensino Superior, Universidade Agostinho Neto - Angola. Director Geral Adjunto Para a Área Académica do Instituto Superior de Ciências de Educação do Huambo. Angola. Correio: vindassimanuel@yahoo.com.br 
João Baptista Machado Sousa; Mário José da Costa Rodrigues; Arlindo Emílio Joaquim Pedro; Afonso Vindassi Manuel

Telos Vol. 20, No. 3 (2018). 450-467

Palavras-Chave: Produção Científica, Investigação Científica, ISCED-Huambo.

RECIBIDO: Mayo 2018

ACEPTADO: Agosto 2018

\section{RESUMEN}

La presente investigación forma parte de un informe sobre la producción científica (PC) de los docentes del Instituto Superior de Ciencias de Educación de Huambo en el período de 2013 a 2017. El objetivo es sistematizar la producción científica que el capital humano de esta institución ha venido a desarrollar en este período. Se Consideró como indicadores para evaluar el Índice de Producción Científica (IPC), el número de artículos publicados en revistas científicas nacionales e internacionales, la participación en eventos científicos nacionales e internacionales y finalmente el número de libros e capítulos de libros publicados. Desde el punto de vista metodológico, se utilizaron tres instrumentos para la recopilación de datos: una encuesta, una guía de entrevista y una guía para el análisis documental con el que se analizaron los documentos existentes en los procesos de los docentes y a este último fue apoyado una búsqueda en diferentes bases de datos y motores en Internet y a las revistas citadas por los encuestados. Al final se utilizó el método de triangulación de fuentes, para contrastar las informaciones obtenidas por los instrumentos antes declarados. Como resultado de este análisis, se constató119 artículos científicos publicados, entre estos 75 en revistas nacionales y 44 internacionales; 189 participaciones en eventos científicos, de las cuales 113 nacionales y 76 internacionales, 7 libros publicados y, finalmente un capítulo del libro.

Palabras clave: Producción Científica, Investigación Científica, ISCED-Huambo.

ABSTRACT
The present research is part of a report on the scientific production (CP) of the
teachers of the Higher Institute of Educational Sciences of Huambo from 2013 to
2017. The objective is to systematize the scientific production that the human capital
of this institution has been in this period. We consider as indicators to evaluate the
Scientific Production Index (CPI), the number of articles published in national and
international scientific journals, participation in national and international scientific
events and finally the number of books published. From a methodological point of
view, three instruments were used to collect data: a survey, an interview guide and a
guide to the documentary analysis that analyzed the documents in the teachers' files,
and the latter was Search on the data bases and the engines on the Internet and the
journal cited by the teachers. In the end, the source triangulation method was used to
contrast the information obtained by the previously declared instruments. As a result
of this analysis, It found 119 published scientific articles, among them 75 in national 
Índice de produção científica dos docentes do Instituto Superior de Ciências de Educação do Huambo

and 44 international journals; 189 participations in scientific events, of which 113 national and 76 international, 7 books published and, finally a book chapter.

Keywords: Scientific Production, Scientific Research, ISCED-Huambo.

\section{Introdução}

Nas últimas duas décadas, a "produção científica" tem constituído a preocupação das Instituições de Ensino Superior de todo o mundo; ao nível internacional identificam-se investigações que vão desde o conceito à origem da produção científica (Piedra Salomón e Martínez Rodríguez 2007; Dal-Soto; Alves e Souza, 2016), a sua análise (Lino; Backes; Ferraz; Reibnitz e Martini, 2010; Caballero Rico; Uresti Marín e Ramírez de León, 2012), os factores que condicionam a productividade nas investigações (Navarro e Lobo, 2017), a avaliação e o seu impacto (Struchiner, 2013; Wood Jr. e Costa, 2015; Tarango; Hernández-Gutiérrez; VázquezGuzmán, 2015; Ortiz Torres, 2015; Barata, 2015), a indexação de trabalhos em bases de dados e a proposição de um índice-h para a produção científica (Dantas, 2004; Santos, 2011; Simioni; Dallacorte; Jacoski, 2016), a estatística de publicações (Artigas e López Juvinao, 2016), e a sua disponibilidade em acesso aberto na África Lusófona (Guambe; Bueno-de-la-fuente, 2013).

Em Angola, esta problemática tem preocupado o capital humano que dirige as Instituições de Ensino Superior (IES), na medida em que constituí um dos indicadores de avaliação para o ranking das universidades. Assim sendo, os autores angolanos consultados reconhecem o fraco investimento que se tem realizado na área da investigação científica, o que resulta na baixa qualidade da produção científica que se realiza nas IES angolanas (Teixeira, 2013; 2015; Ferreira, 2016; Sambo, 2016; Leitão, 2016; Neto, 2016; Da Rocha, 2017).

Estas limitações refletem-se na baixa qualidade do processo docenteeducativo, que se verifica nas universidades, na não acreditação destas instituições pelas agências internacionais e, consequentemente, na ausência das universidades angolanas em lugares de destaque nos ranking internacionais. 
João Baptista Machado Sousa; Mário José da Costa Rodrigues; Arlindo Emílio Joaquim Pedro; Afonso Vindassi Manuel

Telos Vol. 20, No. 3 (2018). 450-467

Como resultado da sistematização teórica realizada pelos autores do presente artigo, relacionada com a problemática exposta nos parágrafos anteriores, adicionado aos objectivos do plano de desenvolvimento institucional (PDI) do Instituto Superior de Ciências de Educação do Huambo, adiante ISCED-Huambo, começou-se a investir primeiramente no capital humano desta instituição com formações de curta duração (diplomados), mestrados e doutoramentos, seguido da criação de um Centro de Estudos e Investigação Científica (CEIC), que tem a responsabilidade de realizar investigações ao nível das ciências de educação e, finalmente na sistematização regular de informações sobre a produção científica realizada pelo seu quadro docente, o que evidenciou resultados significativos.

Os argumentos antes apresentados serviram de motivação para a redação deste artigo, cujo objectivo é sistematizar a produção científica que os docentes do ISCED do Huambo têm realizado, no período de 2013 a 2017.

\section{A produção científica e seu impacto no Ensino Superior}

A literatura especializada sobre a produção científica permitiu identificar diferentes pontos de vista à volta do seu conceito. Entre os autores consultados que têm realizado significativas contribuições destacam-se os seguintes: Jiménez (1992), Menezes e Santos (2001), Witter (2005), Piedra Salomón e Martínez Rodríguez (2007). Os mesmos, apesar de assumirem posições diferentes nas suas abordagens, concordam que a produção científica é realizada normalmente por instituições de formação (universidades) e de investigação (centros de investigação). Consideram ainda que a produção científica diz respeito à publicação de livros, capítulos de livros e artigos, bem como à publicação de trabalhos ou resumos de trabalhos em anais de congressos científicos, entre outros.

Em nossa perspectiva e tendo em conta o regulamento da carreira docente do Ensino Superior angolano, considera-se que a produção científica faz referência a todo trabalho realizado em instituições de investigação científica e fundamentalmente de Ensino Superior e que diz respeito à publicação de livros (didácticos ou científicos), 
Índice de produção científica dos docentes do Instituto Superior de Ciências de Educação do Huambo

sebentas, manuais, capítulos de livros e artigos, bem como à participação e publicação de trabalhos ou resumos de trabalhos em anais de congressos científicos.

Nesta direcção, a necessidade de realização da produção científica ao nível mundial surge entre Janeiro a Maio de 1665, quando países como França e Inglaterra publicaram as suas primeiras revistas científicas onde eram editados relatos das experiências que eram realizadas nas áreas de física, química anatomia e meteorologia (Souza, 2006).

Estes elementos serviram para despertar os gestores das Instituições de Ensino Superior e dos Centros de Investigação de todo o mundo sobre a necessidade de se publicar os resultados das experiências que se realizavam em suas instituições.

Santin; Vanz e Stumpf (2015), consideram que a produção científica das universidades revela a sua capacidade de cumprir uma de suas funções mais fundamentais: a criação de novos conhecimentos.

Por outro lado, Wood Jr. e Costa (2015), relatam a importância da investigação, porém, destacam igualmente a necessidade da sistematização e da divulgação destes conhecimentos produzidos. Afinal, o conhecimento científico não se resume na descoberta de factos e leis novas, mas também em sua publicação. Tratase de obter e comunicar resultados.

A análise qualitativa da produção científica de países em desenvolvimento mostra que por um lado, houve melhoria não apenas na quantidade de publicações, como também na qualidade, aferida pelas citações que receberam estes artigos. Por outro lado, houve um incremento significativo no seu impacto relativo médio, que subiu de 0,52 em 2000 para 0,70 em 2011. Este aumento pode ser devido à crescente colaboração na última década entre os países em desenvolvimento e os desenvolvidos (Scientific Electronic Library Online, 2014).

Nassi-Calò (2015), considera que existem sólidas evidências de que a colaboração em investigação científica seja entre instituições, países ou regiões, aumenta a qualidade, visibilidade e impacto das publicações resultantes. Este 
João Baptista Machado Sousa; Mário José da Costa Rodrigues; Arlindo Emílio Joaquim Pedro; Afonso Vindassi Manuel

Telos Vol. 20, No. 3 (2018). 450-467

fenómeno tem atraído a atenção do capital humano responsável pelas tomadas de decisões, como uma forma de fomentar a excelência nas investigações em várias partes do mundo.

Assim sendo, a Fundação de Amparo à Pesquisa de São Paulo, FAPESP (2010, p.12), ao referir-se sobre os dados do Essential Science Indicators no período 2002-2006, indicam que os Estados Unidos da América, liderou o ranking mundial de publicações científicas, com ampla vantagem em relação ao Japão, que ocupava a $2^{\mathrm{a}}$ posição.

Estudos mais recentes realizados por Zanotto; Haeffner e Guimarães (2016), sobre a colaboração na produção científica no Brasil e no Mundo no período de 2000 a 2015, destacam que neste intervalo foram produzidos 26.405 .178 trabalhos científicos pelos 35 países mais produtores do mundo. Os Estados Unidos da América aparece como o país com maior produção científica com 7.359 .519 investigações realizadas, 131.950 .029 citações e um factor de impacto de 17,9. A seguir aparecem países como China, Alemanha, Inglaterra, Japão, França, Canada, Itália, Espanha, Austrália e Índia. No continente africano, destaca-se a presença da África do Sul com um total de 131.749 investigações realizadas, 1.503 .098 citações e um factor de impacto de 11,4 .

Uma investigação realizada sobre a produção científica dos autores da África Lusófona em revistas internacionais no período de 2002 a 2012, demonstrou que Angola se encontrava em segundo lugar com um total de 380 trabalhos (Guambe; Bueno-de-la-fuente, 2013, p.11).

Nesta perspectiva, a Ministra da Ciência e Tecnologia, no período de (20122017), Cândida Teixeira, afirmou recentemente em comunicado de imprensa que o desempenho de Angola em termos de produção científica observou um aumento de $18.8 \%$ de 1995 a 2013 (Teixeira, 2015).

Contudo, autores como Jiménez (1992), Menezes e Santos (2001), Witter (2005), Piedra Salomón e Martínez Rodríguez (2007), Wood Jr. e Costa (2015), entre 
Índice de produção científica dos docentes do Instituto Superior de Ciências de Educação do Huambo

outros, têm dado significativas contribuições relacionadas com a importância e a necessidade da produção científica.

De modo geral, convergem na necessidade da realização da produção científica pelas Instituições de Ensino Superior e pelos Centros de Investigação Científica e na sistematização e divulgação destes conhecimentos produzidos em revistas científicas especializadas, conferências científicas e livros.

Assim sendo, resulta a sistematização da produção científica do claustro docente do ISCED-Huambo, como um primeiro elemento para contribuir na elaboração de um relatório sobre a produção científica das Instituições de Ensino Superior angolanas.

\section{Metodologia}

Este trabalho enquadra-se nas investigações com enfoque quantitativo (Hernandez Sampieri; Fernandez Collazo e Baptista Lúcio, 2010, p.5), tendo em conta que se pretende quantificar a produção científica dos docentes do ISCED-Huambo no período de 2013 a 2017, como: número de artigos científicos publicados em revistas nacionais e internacionais, a participação em eventos científicos nacionais e internacionais, o número de capítulos de livros e, finalmente, o número de livros publicados.

Para o processo de recolha de dados utilizou-se um inquérito que foi desenhado tendo em conta a escala de Likert, um guia de entrevista e um guia para a análise documental. Todos os instrumentos compostos por cinco indicadores, com os quais se analisou os documentos existentes nos processos dos docentes e a este último foram coadjuvados uma busca em diferentes motores na Internet. No final, utilizou-se o método de triangulação de fontes, para contrastar as informações obtidas pelos instrumentos antes declarados.

Os autores consultados coincidem em suas teorias ao afirmar que na triangulação recolhem-se dados de diferentes vias, combinam-se duas ou mais teorias, fontes de dados ou métodos de investigação no estudo de um fenómeno com o 
João Baptista Machado Sousa; Mário José da Costa Rodrigues; Arlindo Emílio Joaquim Pedro; Afonso Vindassi Manuel

Telos Vol. 20, No. 3 (2018). 450-467

objectivo de compara-los e contrasta-los entre si (Denzin, 1970; Ruiz Aguilera, 1999; Okuda e Gómez-Restrepo, 2005; Vallejo e Franco, 2009).

Estes instrumentos foram aplicados numa amostra total da população constituída por 68 docentes distribuídos pelos cinco departamentos de ensino e investigação que possui o ISCED - Huambo. Os dados recolhidos foram processados, e os resultados apresentam-se nos parágrafos que se seguem.

\section{Resultados e Discussão}

A participação dos docentes aos diferentes eventos científicos, permitiu analisar e interpretar os dados recolhidos e apresentá-los nos seguintes gráficos:

Fig. 1. Gráfico referente à participação em eventos científicos nacionais e internacionais

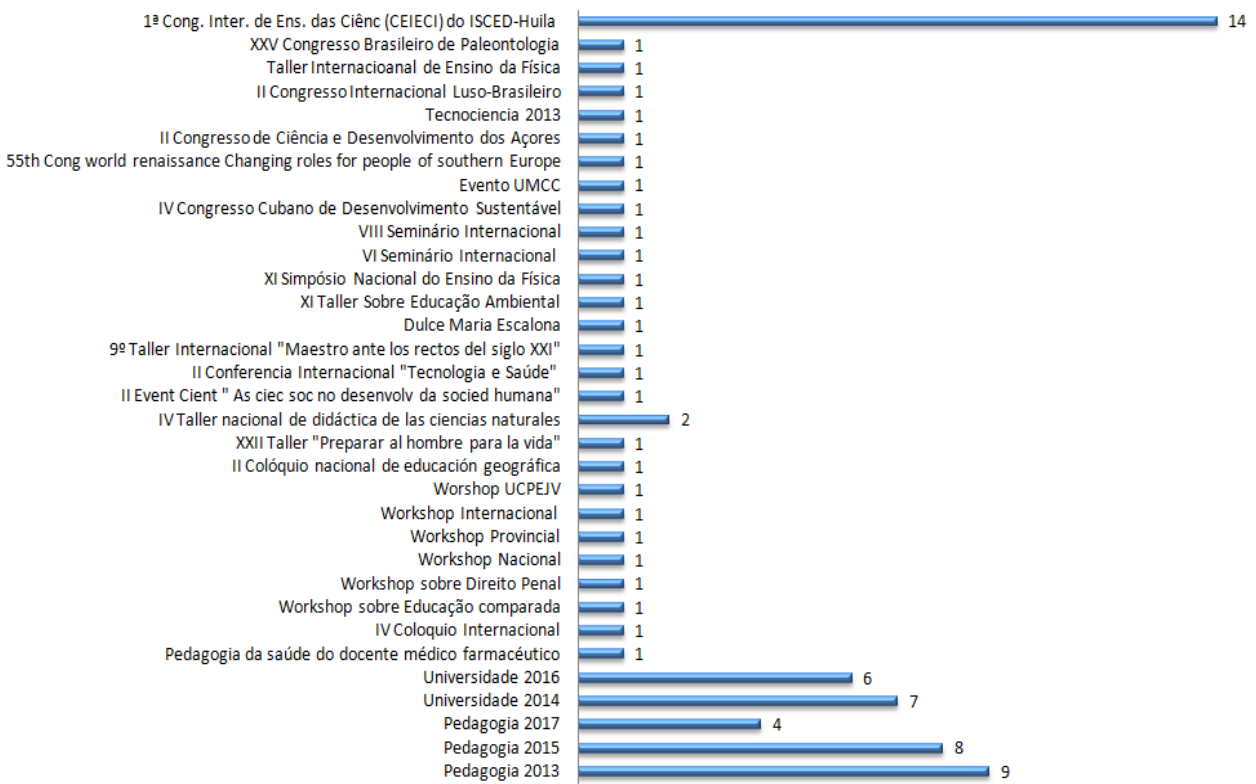

Fonte: elaboração própria.

$\mathrm{Na}$ análise dos dados, referentes aos eventos científicos internacionais, foi possível observar que os docentes do ISCED-Huambo obtiveram uma maior 
Índice de produção científica dos docentes do Instituto Superior de Ciências de Educação do Huambo

participação no I congresso internacional de ensino das ciências, realizado pelo Instituto Superior de Ciências de Educação da Huíla, em Angola com 14 trabalhos apresentados, seguido dos eventos pedagogia 2013 com 9 apresentações, pedagogia 2015 com 8 e universidade 2014 e 2016 com 7 e 6 respectivamente, estes últimos realizados na República de Cuba.

Duque e Quintero (2016), referindo-se à Benítez (2007), consideram que uma das formas mais comuns de troca de experiência e opiniões é com a participação em eventos científicos; um congresso ou jornada científica é uma oportunidade para trocar experiências, êxitos, conhecimento, assim como para discutir dificuldades que os investigadores enfrentam actualmente.

Os autores deste trabalho concordam com estes investigadores e consideram que é necessário incrementar a participação destes em eventos científicos internacionais de Países da mesma região de África e da Lusofonia, como o caso de Brasil e Portugal.

Fig. 2. Gráfico referente à participação em eventos científicos nacionais de 2013 a 2017

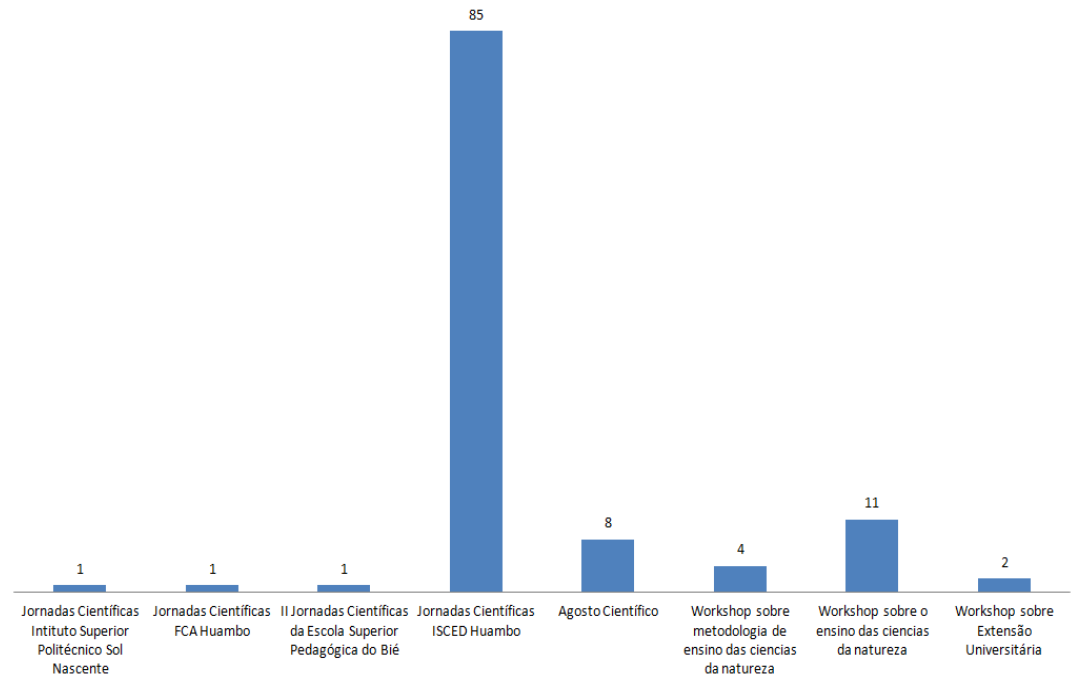

Fonte: elaboração própria. 
João Baptista Machado Sousa; Mário José da Costa Rodrigues; Arlindo Emílio Joaquim Pedro; Afonso Vindassi Manuel

Telos Vol. 20, No. 3 (2018). 450-467

$\mathrm{Na}$ análise dos dados referente à participação em eventos científicos nacionais, é notória a presença dos docentes em actividades que esta instituição (ISCED - Huambo) realiza com 85 participações. Observa-se, desta forma, a necessidade de se melhorar a apresentação de trabalhos em actividades realizadas por outras instituições da mesma região (Angola), sejam públicas ou privadas com o objectivo de se elevar esta dimensão.

Fig. 3. Gráfico referente à publicação de artigos científicos em revistas nacionais e internacionais

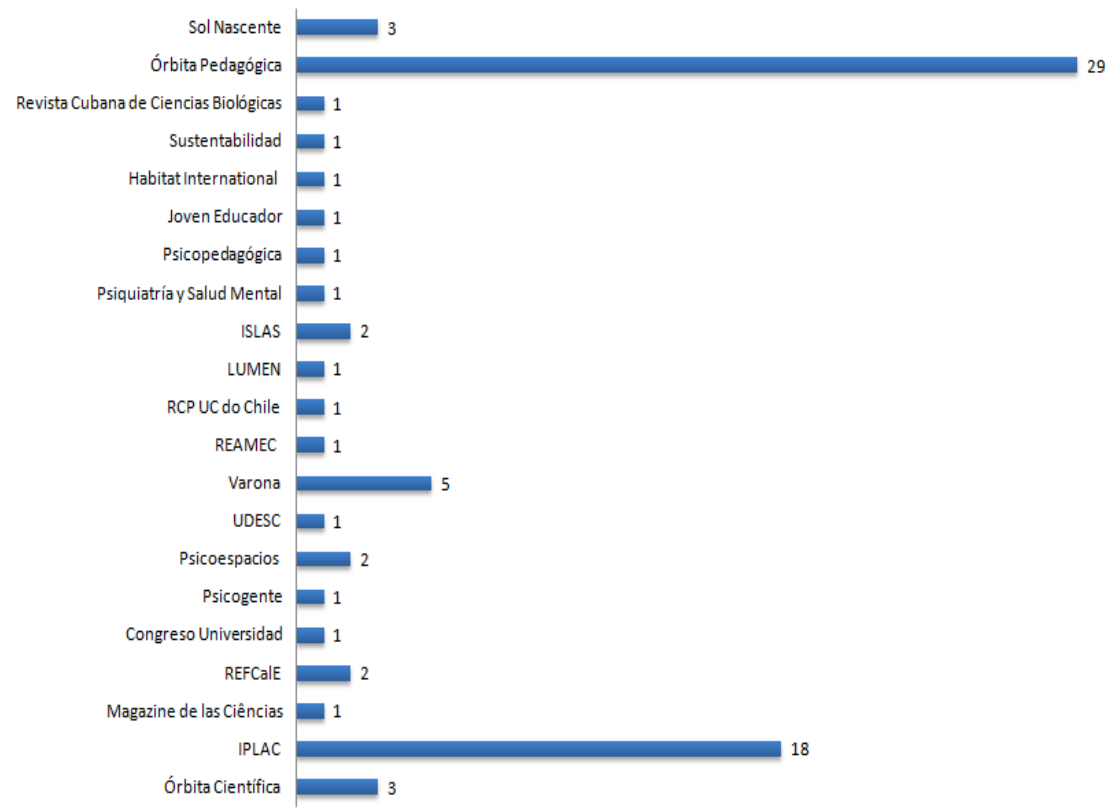

Fonte: elaboração própria.

O gráfico acima representa a publicação de artigos em revistas científicas nacionais e internacionais. É possível observar que os docentes do ISCED-Huambo obtiveram o maior número de publicações na revista Órbita Pedagógica pertencente a instituição onde trabalham (ISCED - Huambo) com 29 artigos, seguido da revista Sol 
Índice de produção científica dos docentes do Instituto Superior de Ciências de Educação do Huambo

Nascente com 3, todas pertencentes a instituições da província do Huambo. Não obstante, recomenda-se incrementar a publicação de artigos em outras revistas.

A análise dos dados, referentes a publicação em revistas científicas internacionais, possibilitou destacar as três com maior número de publicações e observar que estes docentes obtiveram o maior número na revista IPLAC pertencente ao Instituto Pedagógico Latino-americano com 18 trabalhos, seguido da revista "Varona" da Universidade de Ciências Pedagógicas "Enrique José Varona" com 5 e a Órbita científica com 3 artigos publicados.

Entretanto, os resultados mostram que $86.66 \%$ dos artigos foram publicados em revistas não indexadas pelas maiores e mais importantes bases de dados de revistas científicas como: SCOPUS, Scielo, ERIC, ISI ou Web of Science of Institute for Scientific Information, JCR - Journal Citation Reports, REDALYC - Red de Revistas Científicas de América Latina y el Caribe, España y Portugal. Assim sendo, torna-se necessário elevar a qualidade das publicações em redes de revistas com maior visibilidade internacional como as citadas anteriormente.

Alguns estudiosos argumentam que a publicação em revistas científicas é, sem dúvida, uma das principais actividades do investigador, tão importante quanto a própria investigação. Obviamente, se um investigador publica, então torna-se conhecido, suas obras são citadas e dão origem a novos estudos, ele pode até ganhar financiamentos para projectos, elevar o nível de sua investigação e obviamente prestigiar a instituição à qual pertence (Valderrama, 2005).

Tabela 1. Publicação de livros no período de 2015 a 2017.

\begin{tabular}{|l|c|}
\hline \multicolumn{1}{|c|}{ Título do Livro } & \multicolumn{1}{|c|}{$\begin{array}{c}\text { Ano de } \\
\text { Publicação }\end{array}$} \\
\hline & \\
\hline $\begin{array}{l}\text { Fundamentos y principios para la superación en ambientes } \\
\text { virtuales }\end{array}$ & 2015 \\
\hline Preparación del Docente & 2015 \\
\hline
\end{tabular}


João Baptista Machado Sousa; Mário José da Costa Rodrigues; Arlindo Emílio Joaquim Pedro; Afonso Vindassi Manuel

Telos Vol. 20, No. 3 (2018). 450-467

\begin{tabular}{|l|l|} 
Computadores no Ensino 1ra edição & 2016 \\
\hline Elaboração de um Relatório Científico & 2017 \\
\hline Caderno de Exercícios de Pesquisa Operacional & 2017 \\
\hline Aritmética e Teoria dos Números & 2017 \\
\hline Informática Educativa & 2017 \\
\hline & \\
\hline
\end{tabular}

Fonte: elaboração própria.

A tabela acima representa a publicação de livros no período de 2013 a 2017; verifica-se um incremento significativo no ano de 2017, que resulta do investimento dos gestores do ISCED-Huambo sobre o capital humano, por formas a dotá-los de competências (conhecimentos, habilidades e valores) relacionados com a produção científica. A este investimento adiciona-se o esforço que estes docentes têm evidenciado no que diz respeito à investigação científica.

De Souza e Guntzel (2010), realizaram uma investigação sobre a actualidade do ensino superior e a relação entre a investigação e a docência na graduação, onde concluíram que apesar de alguns estudantes valorizarem o professor investigador quando esse sabe aliar os conhecimentos do campo de investigação com os conhecimentos das próprias disciplinas (...) na sua maioria, reconhecem a importância da relação entre o ensino e a investigação, tendo em conta que a postura investigativa acrescenta credibilidade e qualidade ao trabalho docente.

Fig. 4. Gráfico resumido da produção científica

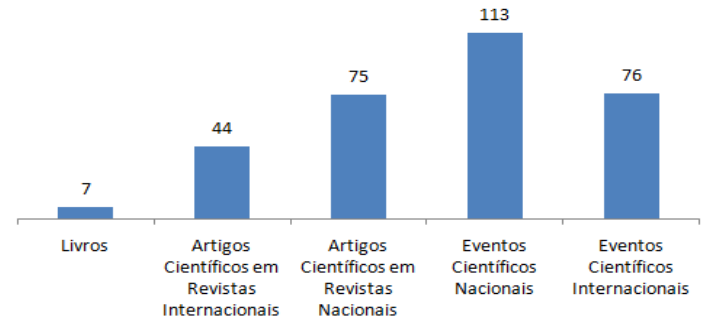

Fonte: elaboração própria 
Índice de produção científica dos docentes do Instituto Superior de Ciências de Educação do Huambo

Em síntese, no gráfico acima apresenta-se o resumo da produção científica realizada pelo claustro docente da instituição supracitada, onde se pode constatar que no período em análise foram publicados 44 artigos em revistas científicas internacionais, 75 em revistas científicas nacionais, 113 participações em eventos científicos nacionais, 76 participações em eventos científicos internacionais e, finalmente, 7 livros entre didácticos a científicos e, finalmente um capítulo de livro. Apesar destes resultados, identificou-se o seguinte:

- Baixa qualidade na produção científica realizada pelos docentes do ISCED-Huambo, o que se manifesta na publicação de $86.66 \%$ destes artigos em revistas com índices baixos e com pouca visibilidade internacional, pelo facto de nenhuma destas revistas encontrarem-se indexadas nas maiores e mais importantes bases de dados como: SCOPUS, Scielo, ERIC, ISI ou Web of Science of Institute for Scientific Information, JCR - Journal Citation Reports, RedAlyc.

- Fraca participação em eventos científicos nacionais realizados por outras instituições e, finalmente, a insuficiente participação em eventos científicos internacionais.

A publicação em revistas indexadas e de alto impacto eleva a visibilidade do investigador e da revista, e consequentemente, melhora o seu factor de impacto (FI). Nesta direcção, Arroyo Mora (2013), assegura que o FI, revela-se de capital importância porque permite valorar de uma maneira relativa, o impacto das investigações na comunidade científica, para além de constituir, ferramenta útil para docentes e investigadores no processo de acreditação e avaliação.

Assim sendo, García Aretio (2015) citando a Buela-Casal (2010), considera que o FI é útil para avaliar a produção científica e a qualidade de uma revista. Porém, actualmente utiliza-se também para avaliar artigos, revistas, docentes, universidades, países, áreas, etc.

Nesta perspectiva, algumas agências financiadoras de projectos utilizam este instrumento (FI) como uma via de decisão para alocação de recursos aos investigadores. 
João Baptista Machado Sousa; Mário José da Costa Rodrigues; Arlindo Emílio Joaquim Pedro; Afonso Vindassi Manuel

Telos Vol. 20, No. 3 (2018). 450-467

\section{Conclusão}

Como resultado da sistematização teórica realizada nos últimos cinco anos, em torno da produção científica que se realiza nas IES, adicionada à recolha e tratamento de dados mediante diferentes instrumentos, apresenta-se as seguintes conclusões:

As transformações que ocorrem ao nível do mundo como resultado dos avanços da ciência e da tecnologia demonstram que as IES angolanas, devem elevar o número de publicações e melhorar a qualidade da produção científica realizada pelo seu claustro docente de modo a alavancar a qualidade do processo docente-educativo, que se verifica nas universidades; favorecer o processo de acreditação destas instituições pelas agências internacionais e, consequentemente, melhorar a posição que ocupam as universidades angolanas nos ranking internacionais;

A análise dos dados sobre a produção científica dos docentes do ISCED-Huambo demonstrou a existência de um compromisso em melhorar a qualidade deste processo nesta instituição, que se manifesta no incremento positivo de publicações realizadas por ano.

Por tanto, face a este incremento na produção científica, nota-se a necessidade de elevar o número de publicações em revistas arbitradas e de alto impacto (Qualis A1 ou Grupo 1), revistas indexadas em bases de dados como: SCOPUS, Scielo, ERIC, ISI ou Web of Science of Institute for Scientific Information, JCR - Journal Citation Reports, REDALYC - Red de Revistas Científicas de América Latina y el Caribe, España y Portugal, por formas a melhorar a visibilidade das investigações realizadas por estes docentes.

\section{Referências bibliográficas}

Artigas, Wileidys e López Juvinao, Danny Daniel. (2016). Estadísticas de publicación de la revista Telos (2013-2015): observando el desempeño editorial. Revista Venezolana de Gerencia. Volume. 21. Número. 76. Venezuela (Pp. 591-605) Arroyo Mora, Daisy. (2013). Importancia de los índices y bases de datos. Universidad de Costa Rica. Disponível em: https://ucrindex.ucr.ac.cr/wp- 
Índice de produção científica dos docentes do Instituto Superior de Ciências de Educação do Huambo

content/uploads/2013/09/\%C3\%8Dndices-y-su-importancia-Daisy-Arroyo-

Mora.pdf. Consulta: 03/08/2018

Barata, Germana. (2015). Em revisão: o impacto da produção científica brasileira para o Brasil. Ciência Cultura. Volume. 67. Número. 4. Brasil. (Pp.06-08).

Caballero Rico, Frida Carmina; Uresti Marín, Rocío Margarita e Ramírez de León, José (2012). Análisis de la producción científica de la Universidad Autónoma de Tamaulipas y evaluación de su impacto en los indicadores educativos de calidad. Revista de la educación superior. Volume. 41. Número. 161. México. (Pp. 31-51).

Da Rocha, Alves. (2017). Entrevista ao Jornal Nova Gazeta. Luanda. Angola

Dal-Soto, Fábio; Alves, Juliano Nunes e Souza, Yeda Swirski de. (2016). A produção científica sobre internacionalização da educação superior na web of science: características gerais e metodológicas. Educ. rev. Volume. 32. Número. 4. Brasil. (Pp. 229-249).

Dantas, Paulo Elias C. (2004). Indexação bibliográfica em bases de dados: O que é? Para que serve? Onde estamos?. Arq. Bras. Oftalmol. Volume. 67. Número. 4. Brasil. (Pp. 569-570).

De Souza, Alba Regina Battisti e Güntzel, Camila. (2010). Qualidade da educação superior e a relação entre pesquisa e docência na graduação: que dizem os estudantes?. O $25^{\circ}$ Simpósio Brasileiro e $2^{\circ}$ Congresso Ibero-Americano de Política e Administração da Educação. São Paulo. Brasil.

Denzin, Norman Kent. (1970). The Research Act in Sociology. Aldine. Chicago.

Duque, Marvella e Quintero, Johana. (2016). Productividad de los docentes Universitarios en la gestión de la investigación científica. REVECITEC. Volume. 6. Número. 2. Venezuela. (Pp. 126-139).

FAPESP (2010). Indicadores de Ciência, Tecnologia e Inovação - Vol. 1, Cap. 4. São Paulo.

Disponível em: http://www.fapesp.br/indicadores/2010/volume1/cap4.pdf. $30 / 08 / 2017$.

Ferreira, Albano (2016). Entrevista ao Jornal Angop. Luanda. Angola. Disponível em: http://aulp.org/node/114345. Consulta: 10/02/2018.

García Aretio, Lorenzo. (2015). Publicar (casi exclusivamente) en revistas de impacto. RIED. Revista Iberoamericana de Educación a Distancia. Volume. 18. Número. 2. Espanha. (Pp. 7-22).

Guambe, Martins Fernando e Bueno-de-la-Fuente, Gema. (2013). Disponibilidade em Acesso Aberto da produção científica da África Lusófona. InCID: Revista de Ciência da Informação e Documentação. Volume. 4. Número. 2. Brasil. (Pp. 5-19).

Hernandez Sampieri, Roberto; Fernández Collazo, Carlos e Baptista Lúcio, Pilar. (2010). Metodología de la investigación. Quinta edición. Editorial McGraw Hill. México. 
João Baptista Machado Sousa; Mário José da Costa Rodrigues; Arlindo Emílio Joaquim Pedro; Afonso Vindassi Manuel

Telos Vol. 20, No. 3 (2018). 450-467

Jiménez de Vargas, Belkys. (1992). Aspectos teóricos sobre la productividad en investigación del docente universitario. Revista Espacios. Volume 13. Número 2. Venezuela. (S/p).

Leitão, Anabela (2016). Entrevista ao portal ciência.ao. Luanda. Angola. Disponível em: $\quad$ http://ciencia.ao/entrevistas/item/658-entrevista-prof-anabela-leitao.

Consulta: 10/02/2018.

Menezes, Ebenezer Takuno e Santos, Thais Helena dos. (2001). Verbete produção técnico-científica. Dicionário Interativo da Educação Brasileira Educabrasil. São Paulo: Midiamix. Disponível em: http://www.educabrasil.com.br/producao-tecnico-cientifica/. Consulta: $19 / 08 / 2017$.

Nassi-Calo, Lilian. (2015). Indicadores bibliométricos da produção científica europeia. SciELO em Perspectiva. Disponível em: http://blog.scielo.org/blog/2015/11/05/indicadores-bibliometricos-da-producaocientifica-europeia/. Consulta: 25/08/2017.

Navarro, Erika Johanna Casanova e Lobo, Maira Alejandra Pino. (2017). Factores que condicionan la productividad en investigación de los docentes de la Institución de Educación Superior Tecnológica FITEC de la ciudad de Bucaramanga. Monografía de Especialización en Pedagogía para la Educación Superior. Universidad Santo Tomas. Colombia.

Neto, Domingos. (2016). Entrevista ao Jornal Nova Gazeta. Luanda. Angola. Disponível em: http://novagazeta.co.ao/?p=12549. Consulta: 15/03/2018.

Okuda Benavides, Mayumi e Gómez-Restrepo, Carlos. (2005). Métodos en investigación cualitativa: triangulación. Revista Colombiana de Psiquiatría. Volume. 34. Número. 1. (Pp. 118-124).

Ortiz Torres, Emilio Alberto. (2015). La evaluación del impacto científico en las investigaciones educativas a través de un estúdio de caso. REDIE. Ensenada. Volume. 17. Número. 2. México. (Pp. 89-100).

Piedra Salomón, Yelina e Martínez Rodríguez, Ailín. (2007). Producción científica. Revista Ciencias de la Información. Volume. 38. Número. 3. Cuba. (Pp.3338).

Ruiz Aguilera, Ariel (1999). Metodología de la Investigación Educativa. Editorial Grifo Chapecó. Bra

Sambo, Maria do Rosário Bragança. (2016). Angola e os desafios para o incremento da produção científica: o financiamento, a cooperação em redes e a avaliação da actividade científica. $6^{\text {a }}$ Conferência FORGES. São Paulo. Brasil

Santin, Dirce Maria; Vanz, Samile Andrea de Souza e Stumf, Ida Regina Chittó. (2015). Internacionalização da produção científica em Ciências Biológicas da UFRGS: 2000-2011. Transinformação. Volume. 27. Número. 3. Brasil. (Pp. 209-218). 
Índice de produção científica dos docentes do Instituto Superior de Ciências de Educação do Huambo

Santos, Gildenir Carolino. (2011). Fontes de indexação para periódicos científicos: um guia para bibliotecários e editores. Editorial E-Color. Brasil.

SCIENTIFIC ELECTRONIC LIBRARY ONLINE. (2014). A bibliometria do mundo em desenvolvimento. SciELO em Perspectiva. Disponível em: http://blog.scielo.org/blog/2014/01/09/a-bibliometria-do-mundo-emdesenvolvimento-publicado-originalmente-na-newsletter-da-elsevier-researchtrends-issue-35-developing-research-in-developing-countries/. Consulta: 25/08/2017.

Simioni, Cinthia Luana; Dallacorte, Caroline e Jacoski, Claudio Alcides. (2016). Proposição de um índice-h para a produção científica da construção civil nacional. Encontros Bibli: revista electrónica de biblioteconomia e ciência da informação. Volume. 21. Número. 46. Brasil. (Pp. 137-153).

Souza, Pereira Salles de. (2006). Publicação de revistas científicas na Internet. Revista Bras Cir Cardiovascular. Volume. 21. Número. 1. Brasil. (Pp. 24-28).

Struchiner, Claudio José. (2013). Avaliação da qualidade da produção científica e suas consequências imprevistas e indesejadas: um conceito autoevidente?. Cad. Saúde Pública. Volume. 29. Número. 9. Brasil. (Pp.1716-1717).

Tarango, Javier; Hernández-Gutiérrez, Perla-Zukey e Vázquez-Guzmán, David (2015). Evaluación de la producción científica en universidades públicas estatales mexicanas (2007-2011) usando análisis de componentes principales. Revista el profesional de la información. Volume. 24. Número. 5. México. (Pp. 567-576).

Teixeira, Cândida. (2013). Entrevista ao Jornal angonotícias. Luanda. Angola. Disponível em:http://www.angonoticias.com/Artigos/item/39819/ainda-hapouca-producao-cientifica-em-angola. Consulta: 10/02/2018

Teixeira, Cândida. (2015). Acto de abertura da $4^{\mathrm{a}}$ Conferência Nacional sobre Ciência e Tecnologia. Luanda. Angola. Disponível em: http://tpa.sapo.ao/noticias/ciencia/producao-cientifica-em-angola-observou-umaumento-de-188-porcento. Consulta: 10/02/2018

Valderrama, José. (2005). Principales aspectos sobre la preparación de un artículo para ser publicado en una revista internacional de corriente principal. Revista Información Tecnológica. Volume 16. Número 2. Chile. (Pp. 3-14).

Vallejo, Ruth e Franco, Mineira Finol. (2009). La triangulación como procedimiento de análisis para investigaciones educativas. REDHECS-URBE. Volume. 7. Número. 4. Venezuela. (Pp.117-133).

Witter, Carla. (2005.) Produção científica e educação: análise de um periódico nacional. En: Witter, G. (Editor). Metaciência e psicología. Brasil. Editora Alinea. (Pp.199-215).

Wood Jr., Thomas e Costa, Caio Cesar de Medeiros. (2015). Avaliação do impacto da produção científica de programas selecionados de pós-graduação em 
João Baptista Machado Sousa; Mário José da Costa Rodrigues; Arlindo Emílio Joaquim Pedro; Afonso Vindassi Manuel

Telos Vol. 20, No. 3 (2018). 450-467

Administração por meio do índice H. Revista de Administração (RAUSP). Volume. 50. Número. 3. Brasil. (Pp.325-337)

Zanotto, Sónia Regina; Haeffner, Cristina e Guimarães, Jorge Almeida. (2016). Colaboração na produção científica no Brasil e no Mundo: um recorte das áreas do Essential Science Indicators. $5^{\circ}$ Encontro Brasileiro de Bibliometria e Cientometria. São Paulo. Brasil. 